\title{
Spontaneous left iliac vein rupture - case report
}

\author{
Mehmet Kalender, Ahmet Nihat Baysal, Oğuz Ugur, Hayat Gökmengil \\ Cardiovascular Surgery Department, Konya Education and Research Hospital, Konya, Turkey
}

\begin{abstract}
Spontaneous iliac vein rupture is an uncommon cause of retroperitoneal haematoma. Most common concomitant pathology reported was deep vein thrombosis or thrombophlebitis and followed by May-Thurner Syndrome. In this particular case, we observed findings parallel to literature.

A 61 -year-old female patient complaining of abdominal and back pain was admitted to the emergency room. She was a smoker and except that medical history revealed only chronic obstructive pulmonary disease. Also, she had no history of trauma. She suffered from sudden left abdominal and back pain without evident cause. On arrival, her vitals were normal. An abdominopelvic computed tomography (CT) showed large haematoma in the lower abdominal cavity. Retroperitoneal exploration revealed large haematoma and left external iliac vein rupture. The vein was repaired primarily. Postoperative follow-up visit at one month after surgery showed that the patient was doing well.

lliac vein rupture should be taken into account in middle-aged or elderly women with acute onset abdominal pain and acute DVT.
\end{abstract}

Key words: haemorrhagic shock, iliac vein, spontaneous rupture

Acta Angiol 2016; 22, I: 20-22

\section{Introduction}

Rupture of the external iliac vein is a very rare condition, but once it occurs, emergency operation is necessary. Main causes are not clear and further investigations are required. To our knowledge, this is a report showing "Unexpected or unusual presentations of a disease" which is spontaneous rupture of the external iliac vein.

\section{Case report}

A 61-year-old female patient complaining of abdominal and back pain was admitted to the emergency room. She was a smoker and except that medical history revealed only chronic obstructive pulmonary disease. In addition, she hadn't any history of trauma. She suffered from sudden left abdominal and back pain without evident cause. On admission, her blood pressure was $110 / 70 \mathrm{~mm} \mathrm{Hg}$, and her pulse rate was 87/min. Physical examination revealed slight abdominal tenderness. There was no leg swelling or leg pain. Blood tests showed anaemia, with haemoglobin of $10.3 \mathrm{mg} / \mathrm{dL}$ and no other abnormal data were found at blood tests. An abdominopelvic computed tomography (CT) showed large haematoma in the lower abdominal cavity (Fig. I) and a varicose left iliac vein with intravascular thrombosis (Fig. 2). Emergency laparotomy was planned. During anaesthesia induction the patient experienced hypotensive shock and when the abdominal cavity was opened, left retroperitoneal bulging mass-like lesion was observed. Retroperitoneal region exploration revealed large haematoma and left external iliac vein rupture. External iliac vein was rounded with tapes. The vein was repaired and low-molecular weight heparin was administered. The patient was discharged on warfarin therapy. During postoperative course, the patient experienced deep vein thrombosis at the level of superficial femoral vein diagnosed with Doppler ultrasonography. The trombus had resolved after two 


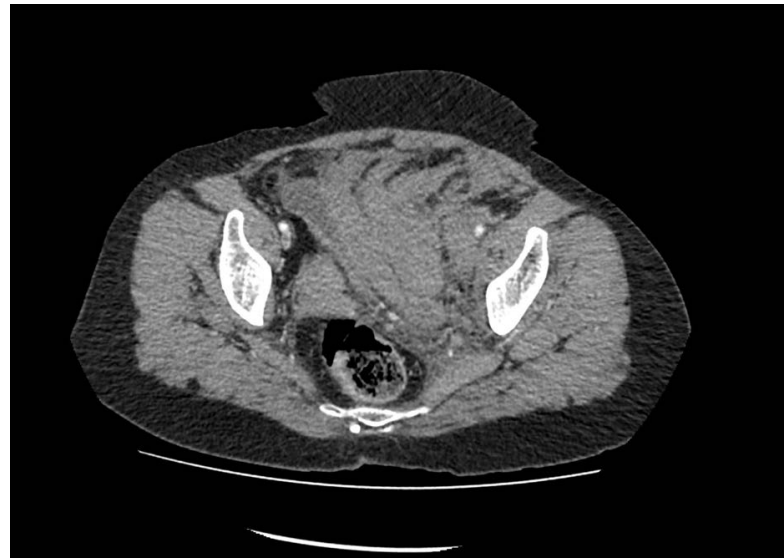

Figure I. Contrast enhanced axial CT images depicted in arterial phase. The left iliac artery is clearly seen but perivascular fat plains are obscured. The left iliac vein is not seen as a separate structure because of haematoma around it

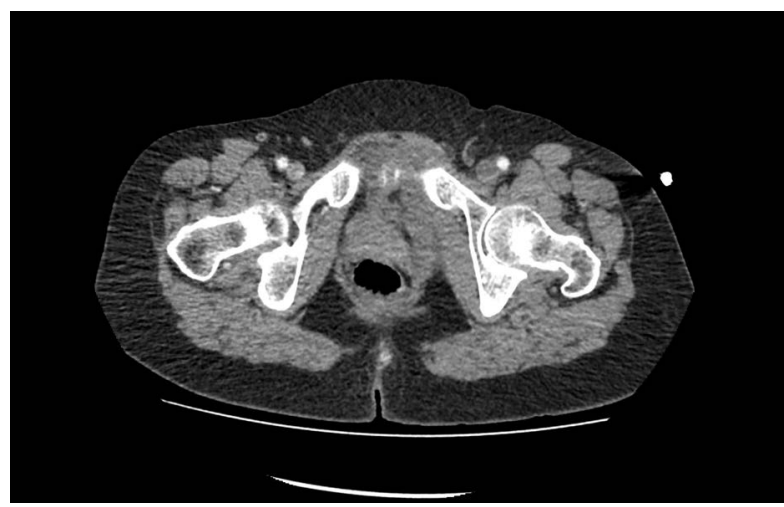

Figure 2. In the lower images present left iliac vein enlargement caused by the abdominal haematoma's mass effect (notice the normal right iliac vein). There is varicose dilatation distal to the rupture

weeks of warfarin treatment and further intervention was not required. Postoperative follow-up visit at one month after surgery showed that the patient was doing well. The patient gave consent to have her information used for scientific publishing.

\section{Discussion}

Spontaneous iliac vein rupture is an uncommon cause of retroperitoneal haematoma. It is mostly reported as case reports [ $1-3]$. The largest series was reported by jiang et al. and it included 9 cases [4]. Most common concomitant pathologies were deep vein thrombosis or thrombophlebitis, followed by May-Thurner Syndrome [4]. Possible aetiological factors have been identified as venous hypertension, as well as the inflammatory venous wall [5-7]. Some oestrogenic factors may explain female preponderance [8]. Our case is similar to literature.
Common clinical characteristics are included sudden onset of left lower abdominal or lumbar pain, swelling of the left lower limb, anaemia, and shock [4]. Our case admitted to emergency department with sudden onset of abdominal pain and laboratory results revealed anaemia. Emergency abdominopelvic CT scan showed aneurysmatic iliac vein and intravascular thrombosis. Patient's haemodynamic condition was deteriorated during anaesthesia induction.

Management options include open surgical procedures, endovascular interventions, combined surgical and endovascular treatment, and conservative therapy. Our patient underwent emergency laparotomy. Open surgical procedures have a drawback such as haemorrhage originating from the vena cava and iliac veins can prove difficult to control. Exposure can be limited both by anatomic location and concurrent venous bleeding, which obscures visualization. Particularly, at the location of the iliac vein, the right iliac artery, can obscure adequate exposure of the venous injury. Also, attempts to gain vascular control by the passage of clamps posterior to a vein can be hazardous, thus risking further injury [9]. Most issues related to the repair of venous injuries pertain to exposure in a haemorrhagic operative field. In trying to obtain vascular isolation for an injury, care should be exercised when trying to pass clamps posterior to venous structures because of the risk of causing further venous injury [10]. At this point combined endovascular and surgical treatment may be considered. Endovascular balloons, in contrast, can be passed intravascularly from a remote location, such as the groin vessels, when the vascular injury site itself may not be visible. Of note, in this scenario, a cut-down of femoral vessels was necessarily performed, given the subsequent aortobifemoral repair, but we expect that this approach would be equally useful through a percutaneous approach [9]. Haemodynamic stability was the key criterion. If haemodynamic variables were stable after resuscitation, the patient was treated conservatively. If there were evidence of further haemorrhage or blue phlegmasia with impending venous gangrene, a surgical procedure was compelled [4].

lliac vein rupture should be taken into account in middle-aged or elderly women with acute-onset abdominal pain and acute DVT.

\section{References}

I. Tannous H, Nasrallah F, Marjani M (2006) Spontaneous iliac vein rupture: case report and comprehensive review of the literature. Ann Vasc Surg; 20: 258-262.

2. Kim YH, Ko SM, Kim HT (2007) Spontaneous rupture of the left common iliac vein associated with May-Thurner syndrome: successful management with surgery and placement of an endovascular stent. Br J Radiol; 80: el 76-179. 
3. Borkon MJ, Dassinger MS 3rd, Dattilo JB (2009) Spontaneous iliac vein rupture. Am Surg; 75: II5I-I I52.

4. Jiang J, Ding X, Zhang G, Su Q, Wang Z, Hu S (2010) Spontaneous retroperitoneal hematoma associated with iliac vein rupture. J Vasc Surg; 52: 1278-1282.

5. Hossne WS, Nahas PS, Vasconcelos E (196I) Spontaneous rupture of the iliac vein: acute abdomen. Arq Circ Clin Exp; 24: 27-30.

6. Herczeg B, Karpathy L, Kovecs G et al (1967) Spontaneous rupture of the iliac vein with lethal hemorrhage. Zentralbl Chir; 92 552-555.
7. McDonald RT, Vorpahl TE, Caskey J (1980) Spontaneous rupture of the iliac vein. Vasc Surg; 14: 330-333.

8. Chen X-R, Zhang W-Z, Xin D-Y (2013) Spontaneous rupture of the left external iliac vein: case report. Surg Science; 4: 325-328.

9. Tillman BW, Vaccaro PS, Starr JE, Das MB (2006) Use of an endovascular occlusion balloon for control of unremitting venous hemorrhage. J Vasc Surg; 43 (2): 399-400.

10. Mullins RJ, Huckfeldt R, Trunkey D (1996) Abdominal vascular injuries. Complex and challenging problems in trauma surgery. Surg Clin North Am; 76: 8I3-832. 